\title{
Night sleep duration and risk of each lipid profile abnormality in a Chinese population: a prospective cohort study
}

\author{
Qiaofeng Song ${ }^{1 \dagger}$, Xiaoxue $\mathrm{Liu}^{1 \dagger}$, Wenhua Zhou', Shouling $\mathrm{Wu}^{2^{*}}$ and Xizhu Wang ${ }^{1 *}$
}

\begin{abstract}
Background: To explore the associations between sleep duration and abnormalities in serum lipid levels in a Chinese population.

Methods: A prospective study was conducted with 34,260 participants from the general Chinese population. Sleep duration was categorized as $\leq 5,6,7,8$ or $\geq 9 \mathrm{~h}$. Each lipid profile abnormality was defined according to the Chinese Guidelines for the Prevention and Treatment of Dyslipidemia in Adults (2016). The Cox proportional hazards model was used to assess the association between sleep duration and dyslipidemia.

Results: Compared with a $7 \mathrm{~h}$ sleep duration, long sleep duration ( $\geq 9 \mathrm{~h})$ was significantly associated with low highdensity lipoprotein cholesterol (HDL-C) levels (hazard ratio (HR): 1.24; $95 \%$ Cl: 1.12-1.38). In subgroup analyses, the positive association between long sleep duration and low HDL-C level in men and in the different age groups was more pronounced than the association in women. No significant interactions were observed in the association between sleep duration and each abnormal serum lipid level by sex/age in the study population (P-interaction> 0.05).
\end{abstract}

Conclusions: These findings suggest that long sleep duration is associated with low HDL-C level among the Kailuan community population.

Keywords: Sleep duration, Dyslipidemia, High-density lipoprotein cholesterol, Low-density lipoprotein cholesterol, Prospective cohort

\section{Introduction}

Short or long sleep durations have been reported to be associated with a higher risk of diabetes mellitus [1-3], obesity $[4,5]$, hypertension [6, 7], cardiovascular disease (CVD) [8, 9] and atherosclerosis [10]. In fact, the mechanisms underlying these relationships are still unclear. In recent years, several studies have examined the links

\footnotetext{
*Correspondence: drwusl@163.com; tsrmyy_wxz@126.com

${ }^{\dagger}$ Qiaofeng Song and Xiaoxue Liu contributed equally to this work.

${ }^{2}$ Department of Cardiology, Kailuan Hospital, North China University of Science and Technology, Tangshan 063000, China

'Department of Cardiology, Tangshan People's Hospital, North China University of Science and Technology, No.65 Shengli Road, Lunan District, Tangshan 063000, China
}

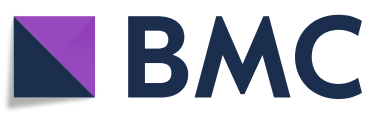

(c) The Author(s). 2020 Open Access This article is licensed under a Creative Commons Attribution 4.0 International License, which permits use, sharing, adaptation, distribution and reproduction in any medium or format, as long as you give appropriate credit to the original author(s) and the source, provide a link to the Creative Commons licence, and indicate if changes were made. The images or other third party material in this article are included in the article's Creative Commons licence, unless indicated otherwise in a credit line to the material. If material is not included in the article's Creative Commons licence and your intended use is not permitted by statutory regulation or exceeds the permitted use, you will need to obtain permission directly from the copyright holder. To view a copy of this licence, visit http://creativecommons.org/licenses/by/4.0/. The Creative Commons Public Domain Dedication waiver (http://creativecommons.org/publicdomain/zero/1.0/) applies to the data made available in this article, unless otherwise stated in a credit line to the data. between sleep duration and risk factors for CVD, including lipids [11-21]. Dyslipidemia is characterized by high levels of total cholesterol (TC), low-density lipoprotein cholesterol (LDL-C), triglycerides (TG) and low levels of high-density lipoprotein cholesterol (HDL-C), and it increases the risk of CVD $[22,23]$ and hence remains an important issue in the field of health promotion and disease prevention. To prevent CVD, it is important to identify and improve the risk factors (including sleep duration) associated with serum TG, HDL-C or LDL-C levels.

Increasing evidence has suggested that short or long sleep durations might be associated with serum lipid 
profiles [11-21]. However, the findings have been inconsistent. Cross-sectional associations have been found between short sleep duration and lower HDL-C levels in adult American women with type 2 diabetes [19] and adult Japanese women from the general population [11]. A recent analysis of 2705 participants from the National Health and Nutrition Examination Survey showed that short sleep duration was associated with low HDL cholesterol [24]. In contrast, long sleep durations were found to be associated with low HDL$\mathrm{C}$ levels among a Korean adult population from the Korean National Health and Nutrition Examination Survey [16]. A survey from the Coronary Artery Risk Development Study involving 503 black and white adults aged 32-51 years showed that long sleep duration was associated with increased future TC levels and TG levels but not with HDL-C [15]. However, a common limitation to these published studies is the lack of parameters related to snoring status and lack of a large sample cohort study. Little is known about the longitudinal relationships among factors. Based on this background, this study from Kailuan aimed to explore the association between sleep duration and each lipid profile abnormality stratified by age and sex.

\section{Methods}

\section{Study design and participants}

The Kailuan study was a prospective cohort study involving 101,510 participants (men: 81,110; women: 20, 400; ages: $18-98$ years) in the Kailuan community from June 2006 to October 2007 (visit 1) [25]. The participants were employees (including the retired) of Kailuan (Group) Co. Ltd. that represent all levels of the society, and they are employed as coalminers, administrators, secretaries, accountants, as well as the supportive and service staff, such as policemen, doctors, nurses, vendors, teachers. Of the 101,510 people who participated in the survey, 34,260 adults were included in the analysis after excluding the following participants: 62,200 subjects who had a history of dyslipidemia, 1436 subjects with missing information about baseline sleep duration or serum lipid profiles, and 3614 subjects without data for 2008-2009 (visit 1), 2010-2011 (visit 2), 2012-2013 (visit 3), and 2014-2015 (visit 4) follow-up visits (Fig. 1). The health interview survey was performed using selfadministered structured questionnaires to obtain information on sociodemographic characteristics, health status, and health behaviors. Before the study, all doctors and nurses received rigorous unified training.

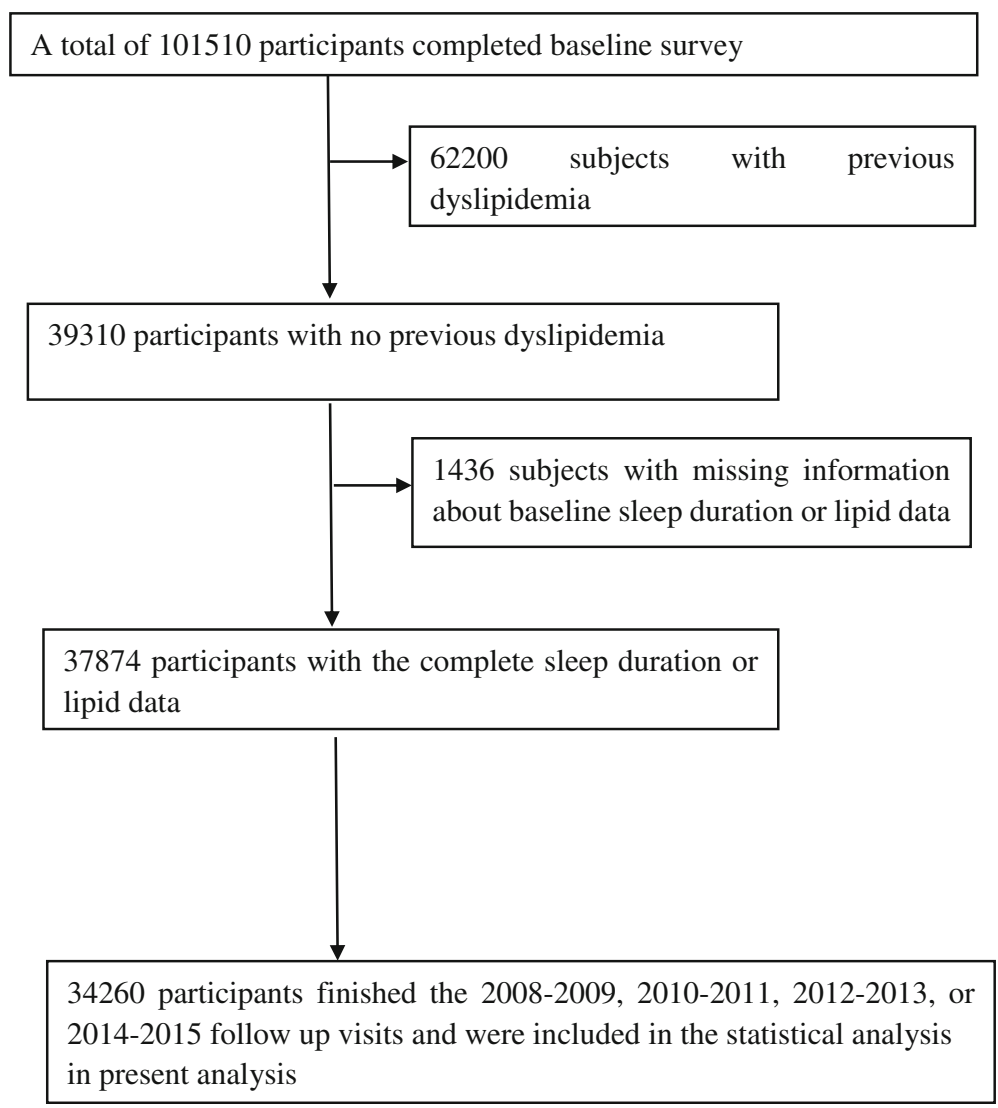

Fig. 1 Flow chart of the present study 


\section{Assessment of sleep duration}

Sleep duration data were collected via self-reported answers to the question "How many hours of sleep have you had on an average at night in the preceding 3 months?" We divided sleep durations into five groups according to the responses: $\leq 5 \mathrm{~h}, 6 \mathrm{~h}, 7 \mathrm{~h}, 8 \mathrm{~h}$, and $\geq 9 \mathrm{~h}$. Additionally, participants were asked to answer "yes" or "no" to the question "Do you generally snore when you sleep?" [25, 26].

\section{Assessment of potential covariates}

The clinical examination consisted of a medical history, a physical examination, anthropometric measurements, and self-administered questionnaires on lifestyle characteristics [25, 27], such as sleep duration,regular leisuretime physical activity, smoking habits, and dailyalcohol consumption. Physical activity was classified as $\geq 4$ times per week and $\geq 20 \mathrm{~min}$ at a time (active physical activity), $<80$ min per week, or none. Smoking status and drinking status were classified as never, former, or current according to the self-reported information. The questionnaire information was double-entered. The body mass index (BMI) was calculated as the weight (kg) divided by the height squared $\left(\right.$ meters $^{2}$ ). Blood pressure was measured three times using a standardized sphygmomanometer while the participant was in a seated position. The average of the three measurements was used in this study.

Blood samples were drawn by trained phlebotomists from participants after an overnight fast for 8-12 h. Blood indicators, including TG, TC, LDL-C, HDL-C, fasting blood glucose (FBG), and high-sensitivity Creactive protein (h-CRP) levels, were measured. TC and TG were measured enzymatically. HDL-C and LDL-C levels were measured using the direct test method [28]. All blood samples were analyzed using a Hitachi 747 autoanalyzer (Hitachi; Tokyo, Japan) [25]. All data was directly uploaded to the oracle database. Diabetes was defined as having a history of diabetes, the use of glucose-lowering agents, or an FBG $\geq 7 \mathrm{mmol} / \mathrm{l}$. Hypertension was defined as a systolic blood pressure $\geq 140$ $\mathrm{mmHg}$, a diastolic blood pressure $\geq 90 \mathrm{mmHg}$, a history of hypertension and/or the use of antihypertensive agents.

\section{Diagnosis of each lipid profile abnormality}

Each lipid profile abnormality was defined according to the Chinese guidelines on the prevention and treatment of dyslipidemia in adults (2016) [29]. Specifically, abnormal TC was defined as TC $\geq 5.2 \mathrm{mmol} / \mathrm{L}$, abnormal TG were defined as TG $\geq 1.70 \mathrm{mmol} / \mathrm{L}$, abnormal LDL- $\mathrm{C}$ was defined as $\mathrm{LDL}-\mathrm{C} \geq 3.4 \mathrm{mmol} / \mathrm{L}$, and abnormal HDL-C was defined as HDL-C $<1.0 \mathrm{mmol} / \mathrm{L}$.

\section{Statistical analyses}

Continuous variables were described by their means \pm standard deviations, and groups were compared using a one-way analysis of variance (ANOVA). Categorical variables were described as percentages and compared using the Chi-square test. The Cox proportional hazards model was used to estimate the HR for the incidence of each lipid profile abnormality in relation to sleep duration and baseline covariates. The follow-up time was calculated from the 2006 interview to the date when lipid profile abnormalities were detected, date of death, or date of the last attended interview in this analysis, whichever came first. For all models, the adequacy of the Cox proportional hazards model was assessed. Model 1 was adjusted for age and sex. Model 2 was further adjusted for the level of education, smoking, alcohol consumption, physical activity, and snoring. Model 3 was further adjusted for hypertension, diabetes mellitus, BMI, and h-CRP. Because transient changes in lipid profile (e.g., reversion from dyslipidemia to normal blood lipid) can impact the current results, the above analysis was repeated after excluding individuals with these conditions (Table 3, sensitivity analysis). To investigate whether age/sex acted as an effect modifier in the relationship between sleep duration and dyslipidemia, a regression model with an interaction term between age/ sex and sleep duration was used. The statistical analysis was performed using SAS 9.4. All statistical tests were two-sided, and the significance level was set at 0.05.

\section{Results}

The baseline characteristics of the study participants by sleep duration are shown in Table 1. The mean age of the participants was 49.13 years (range 20-97 years), and $76.26 \%$ were men. The percentages of participants who reported sleeping for $\leq 5 \mathrm{~h}, 6 \mathrm{~h}, 7 \mathrm{~h}, 8 \mathrm{~h}$, and $\geq 9 \mathrm{~h}$ per night were 6.07, 15.72, 16.42, 60.05, and $1.74 \%$, respectively. Age, sex, education, smoking, alcohol consumption, physical activity, hypertension, diabetes mellitus, and snoring symptoms differed between the sleep duration categories.

Table 2 shows the characteristics of the study participants by dyslipidemia status. Participants with dyslipidemia were more likely to be men, have less than a high school education, be current smokers, be current drinkers, have a higher h-CRP level, have a higher blood pressure, have a higher prevalence of snoring symptoms, be overweight and have diabetes.

The association between sleep duration and each abnormal serum lipid level was examined using multivariate Cox regression models. After adjusting for covariates, the long sleep duration $(\geq 9 \mathrm{~h})$ [16] was significantly associated with low HDL-C levels (HR: 1.24; 95\% CI: $1.12-1.38)$ compared with a $7 \mathrm{~h}$ sleep duration 
Table 1 Baseline characteristics according to sleep duration

\begin{tabular}{|c|c|c|c|c|c|c|}
\hline \multirow[t]{2}{*}{ Variable } & \multicolumn{5}{|c|}{ Sleep duration (h) } & \multirow[t]{2}{*}{$P$ value } \\
\hline & $\begin{array}{l}\leq 5 \\
(n=2081)\end{array}$ & $\begin{array}{l}6.0 \\
(n=5387)\end{array}$ & $\begin{array}{l}7 \\
(n=5624)\end{array}$ & $\begin{array}{l}8.0 \\
(n=20,573)\end{array}$ & $\begin{array}{l}\geq 9 \\
(n=595)\end{array}$ & \\
\hline \multicolumn{7}{|l|}{ Questionnaire-based data } \\
\hline Age, years & $54.18 \pm 12.29$ & $51.38 \pm 12.45$ & $48.59 \pm 12.89$ & $48.27 \pm 12.61$ & $46.11 \pm 14.91$ & $<0.001$ \\
\hline Male, n (\%) & $1741(83.66)$ & $4510(83.72)$ & 4396(78.17) & $15,052(73.16)$ & $427(71.76)$ & $<0.001$ \\
\hline High school or above, n (\%) & 386(18.55) & 1337(24.82) & $1941(34.51)$ & 3656(17.77) & 222(37.31) & $<0.001$ \\
\hline Current smoker, n (\%) & $1148(55.17)$ & $3016(55.99)$ & 2949(52.44) & 4883(23.73) & $299(50.25)$ & $<0.001$ \\
\hline Current alcohol consumption, n (\%) & $1127(54.16)$ & $3167(58.79)$ & $3183(56.60)$ & $5097(24.78)$ & 304(51.09) & $<0.001$ \\
\hline Physically active, $n(\%)$ & $556(26.72)$ & $1216(22.57)$ & 1186(21.09) & 1647(8.01) & 110(18.49) & $<0.001$ \\
\hline Snoring, n (\%) & $458(22.01)$ & 976(18.12) & $891(15.84)$ & $1260(6.12)$ & $86(14.45)$ & $<0.001$ \\
\hline \multicolumn{7}{|l|}{ Exam-based data } \\
\hline Hypertension, n (\%) & $811(38.97)$ & 1852(34.38) & 1758(31.26) & 7496(36.44) & 163(27.39) & $<0.001$ \\
\hline Systolic blood pressure, mmHg & $128.70 \pm 20.54$ & $127.29 \pm 20.04$ & $125.14 \pm 19.74$ & $126.93 \pm 20.16$ & $123.01 \pm 20.88$ & $<0.001$ \\
\hline Diastolic blood pressure, mmHg & $81.68 \pm 11.23$ & $81.13 \pm 10.96$ & $80.31 \pm 10.97$ & $81.85 \pm 11.46$ & $78.75 \pm 11.42$ & $<0.001$ \\
\hline Diabetes mellitus, n (\%) & 148(7.11) & $315(5.85)$ & 288(5.12) & 1016(4.94) & $38(6.39)$ & $<0.001$ \\
\hline Fasting blood glucose, $\mathrm{mmol} / \mathrm{L}$ & $5.27 \pm 1.38$ & $5.21 \pm 1.30$ & $5.19 \pm 1.20$ & $5.22 \pm 1.26$ & $5.23 \pm 1.42$ & 0.915 \\
\hline Total cholesterol, mmol/L & $4.37 \pm 0.59$ & $4.37 \pm 0.57$ & $4.35 \pm 0.57$ & $4.39 \pm 0.57$ & $4.29 \pm 0.63$ & $<0.001$ \\
\hline Triglycerides, mmol/L & $0.98 \pm 0.34$ & $0.98 \pm 0.33$ & $0.97 \pm 0.34$ & $1.01 \pm 0.33$ & $0.95 \pm 0.35$ & $<0.001$ \\
\hline Low-density lipoprotein, mmol/L & $2.14 \pm 0.64$ & $2.14 \pm 0.64$ & $2.15 \pm 0.62$ & $2.08 \pm 0.68$ & $2.01 \pm 0.67$ & $<0.001$ \\
\hline High-density lipoprotein, mmol/L & $1.55 \pm 0.36$ & $1.55 \pm 0.33$ & $1.52 \pm 0.32$ & $1.55 \pm 0.31$ & $1.53 \pm 0.32$ & $<0.001$ \\
\hline Body mass index, $\mathrm{kg} / \mathrm{m}^{2}$ & $24.11 \pm 3.35$ & $24.23 \pm 3.32$ & $24.25 \pm 3.37$ & $24.29 \pm 3.37$ & $24.04 \pm 3.51$ & 0.082 \\
\hline High-sensitivity C-reactive protein, mg/L & $0.66(0.28-1.80)$ & $0.64(0.25-1.70)$ & $0.70(0.29-1.70)$ & $0.65(0.22-1.87)$ & $0.80(0.27-2.00)$ & 0.088 \\
\hline
\end{tabular}

(Table 3, Fig. 2). Moreover, the association between long sleep duration and the risk of low HDL-C levels remained significant even after excluding individuals with transient changes in lipid profile from the analysis (HR: 1.21; 95\% CI: 1.07-1.35) (Table 3).

Table 4 shows the association between sleep duration and each abnormal serum lipid level stratified by sex and age. Among men, there was a positive association between long sleep duration and low HDL-C levels (HR: 1.25; 95\% CI: 1.12-1.41) in the fully adjusted model. Conversely, among women, there was a nonsignificant positive association between long sleep duration and low HDL-C levels in the fully adjusted model. A positive association between long sleep duration and low HDL-C levels was found in participants aged < 60 years (HR: 1.24; 95\% CI: 1.10-1.39) but not participants aged $\geq 60$ years (HR: 1.12; 95\% CI: 0.90-1.40). However, no significant interactions were observed in the association between sleep duration and each abnormal serum lipid level by sex/age in the study population (P-interaction $>0.05$ ).

\section{Discussion}

This prospective study demonstrated that long sleep duration was associated with an increased risk of future low HDL-C level. This association was observed independent of age, sex, BMI, h-CRP, smoking habits, alcohol consumption, physical activity, snoring, education, diabetes mellitus, and hypertension. However, no associations were revealed between sleep duration and the risk of future high TG, TC, or LDL$\mathrm{C}$ levels. A sensitivity analyses further confirmed these findings.

Several studies have reported the associations between serum lipid profiles and sleep duration, although the results of these studies have been inconsistent $[11-13,18,20,30]$. A National Health and Nutrition Survey in Japan showed a U-shaped $(\leq 5 \mathrm{~h}, \geq 9 \mathrm{~h})$ relationship between sleep duration and a high level of TG and between sleep duration and a low level of HDL-C [11]. Consistent with this finding, a study from the China Health and Nutrition Survey (2009) involving 8574 adults showed that both shorter $(\leq 6 \mathrm{~h})$ and longer $(\geq 10 \mathrm{~h})$ sleep durations were associated with higher risks of abnormal serum lipid profiles [18]. However, two cross-sectional studies conducted in the USA and Japan showed that selfreported sleep duration was not associated with hypercholesterolemia [20,30]. On the other hand, the Kansai Healthcare Study in Japan reported that 
Table 2 Comparisons between patients with and without dyslipidemia in the Kailuan study

\begin{tabular}{|c|c|c|c|}
\hline \multirow[t]{2}{*}{ Variable No. } & \multirow{2}{*}{$\begin{array}{l}\text { Dyslipidemia } \\
n=22,011\end{array}$} & \multirow{2}{*}{$\begin{array}{l}\text { Without dyslipidemia } \\
n=12,249\end{array}$} & \multirow[t]{2}{*}{$P$ value } \\
\hline & & & \\
\hline \multicolumn{4}{|l|}{ Questionnaire-based data } \\
\hline Age, years & $48.45 \pm 12.18$ & $50.36 \pm 13.69$ & $<0.001$ \\
\hline Male, n (\%) & $16,683(75.79)$ & $9443(77.09)$ & $<0.05$ \\
\hline Female, n (\%) & $5328(24.21)$ & $2806(22.91)$ & \\
\hline High school or above, n (\%) & 4982(22.63) & $2560(20.90)$ & $<0.001$ \\
\hline Current smoker, n (\%) & $8162(37.08)$ & 4133(33.74) & $<0.001$ \\
\hline Current alcohol consumption, n (\%) & 8606(39.10) & $4272(34.88)$ & $<0.001$ \\
\hline Physically active, n (\%) & 2989(13.58) & 1726(14.09) & 0.210 \\
\hline Snoring, n (\%) & 2479(11.26) & 1192(9.73) & $<0.001$ \\
\hline \multicolumn{4}{|l|}{ Exam-based data } \\
\hline Hypertension, n (\%) & 7834(35.59) & $4246(34.66)$ & 0.08 \\
\hline Systolic blood pressure, $\mathrm{mmHg}$ & $126.81 \pm 19.81$ & $126.58 \pm 20.69$ & $<0.001$ \\
\hline Diastolic blood pressure, mmHg & $81.70 \pm 11.23$ & $80.92 \pm 11.42$ & $<0.001$ \\
\hline Diabetes mellitus, n (\%) & 1215(5.52) & $590(4.82)$ & $<0.001$ \\
\hline Fasting blood glucose, mmol/L & $5.25 \pm 1.27$ & $5.156 \pm 1.26$ & $<0.001$ \\
\hline Total cholesterol, $\mathrm{mmol} / \mathrm{L}$ & $4.44 \pm 0.56$ & $4.26 \pm 0.57$ & $<0.001$ \\
\hline Triglycerides, mmol/L & $1.04 \pm 0.33$ & $0.92 \pm 0.32$ & $<0.001$ \\
\hline Low-density lipoprotein, mmol/L & $2.14 \pm 0.67$ & $2.03 \pm 0.65$ & $<0.001$ \\
\hline High-density lipoprotein, mmol/L & $1.54 \pm 0.32$ & $1.57 \pm 0.32$ & $<0.001$ \\
\hline Body mass index, $\mathrm{kg} / \mathrm{m}^{2}$ & $24.54 \pm 3.35$ & $23.74 \pm 3.32$ & $<0.001$ \\
\hline High-sensitivity C-reactive protein, mg/L & $0.70(0.27-1.90)$ & $0.58(0.20-1.58)$ & $<0.001$ \\
\hline
\end{tabular}

moderate $(5-7 \mathrm{~h})$ and/or long $(\geq 7 \mathrm{~h})$ sleep durations decreased the risk of future low HDL-C and high TG levels [12]. Additionally, Juliana C. Chan et al. concluded that long $(>9.25 \mathrm{~h})$ sleep duration was associated with lower risks of high TC and LDL-C in children and adolescents [13]. These prospective findings suggest that long sleep duration is associated with low HDL-C levels among the Chinese population. Differences in the age distribution of the study population, lifestyle, socioeconomic status, incomplete control for confounding factors, or different categorization and cutoff points of sleep duration may explain the inconclusive associations thus far.

Several studies have found sex differences in the associations between sleep duration and abnormal serum lipid levels $[17,18]$. Results from the National Health Interview Survey 2008 found sex differences in the association between sleep duration and hypercholesterolemia, with a positive association found between sleep duration $\leq 5 \mathrm{~h}$ and hypercholesterolemia in women and an inverse association found between sleep duration $\geq 8 \mathrm{~h}$ and hypercholesterolemia in men [17]. Data from the China Health and Nutrition Survey (2009) showed that both short and long sleep durations were associated with higher risks of abnormal serum lipid profiles in women but not in men [18]. However, the present study found no significant interaction between sleep duration and sex with respect to abnormal serum lipid levels and showed that differences occurred in the sleep duration pattern between men and women. However, it is unclear whether these differences induce sex differences in the association between sleep duration and abnormal serum lipid levels. Additionally, the current study found no significant interaction between sleep duration and age with respect to abnormal serum lipid levels. Further research is needed to investigate the association between sleep duration and serum lipid levels.

Moreover, the biological mechanism responsible for the association between long sleep duration and a low HDL-C level is not easily explained. First, several previous studies have shown that prolonged sleep duration may be associated with obesity [31], hypertension [6], diabetes and glucose intolerance [32, 33]. Second, sleep duration and abnormal lipids often share some common risk factors, such as smoking [34], alcohol consumption [35], and lower socioeconomic status [36]. Third, inflammation is one of the most important biological pathways because long sleep durations can lead to increased levels of inflammatory markers. This study also found that the 
Table 3 Model results for the associations between sleep duration and each abnormal serum lipid level

\begin{tabular}{|c|c|c|c|c|c|c|c|c|}
\hline $\begin{array}{l}\text { Sleep } \\
\text { duration } \\
\text { (h) }\end{array}$ & Event, n(\%) & $\begin{array}{l}\text { Model } 1 \\
\mathrm{HR}(95 \% \mathrm{Cl})\end{array}$ & $\begin{array}{l}\boldsymbol{P} \\
\text { value }\end{array}$ & $\begin{array}{l}\text { Model } 2 \\
\mathrm{HR}(95 \% \mathrm{Cl})\end{array}$ & $\begin{array}{l}\boldsymbol{P} \\
\text { value }\end{array}$ & $\begin{array}{l}\text { Model } 3 \\
\text { HR(95\% CI) }\end{array}$ & $\begin{array}{l}\boldsymbol{P} \\
\text { value }\end{array}$ & $\begin{array}{l}\text { Sensitivity analysis } \\
\text { HR(95\% Cl) }\end{array}$ \\
\hline \multicolumn{9}{|l|}{$\mathrm{TC}$} \\
\hline$\leq 5$ & $902(43.34)$ & $0.95(0.88-1.02)$ & 0.170 & 0.95(0.88-1.02) & 0.174 & 0.96(0.89-1.04) & 0.331 & $0.99(0.90-1.10)$ \\
\hline 6.0 & 2397(44.50) & $1.00(0.94-1.06)$ & 0.945 & $1.00(0.94-1.05)$ & 0.893 & $1.00(0.95-1.06)$ & 0.901 & $1.06(0.98-1.14)$ \\
\hline 7.0 & $2520(44.81)$ & reference & & reference & & reference & & reference \\
\hline 8.0 & $8741(42.49)$ & $0.93(0.89-0.97)$ & 0.001 & $0.99(0.94-1.03)$ & 0.527 & $0.99(0.94-1.04)$ & 0.647 & $1.06(0.99-1.13)$ \\
\hline$\geq 9$ & 237(39.83) & $0.92(0.80-1.05)$ & 0.216 & $0.92(0.81-1.05)$ & 0.230 & $0.93(0.81-1.06)$ & 0.278 & $0.98(0.83-1.16)$ \\
\hline \multicolumn{9}{|l|}{ TG } \\
\hline$\leq 5$ & $555(26.67)$ & $0.97(0.88-1.06)$ & 0.495 & $0.96(0.87-1.05)$ & 0.373 & 0.99(0.90-1.09) & 0.795 & $1.03(0.91-1.17)$ \\
\hline 6.0 & 1616(30.00) & $1.04(0.97-1.11)$ & 0.281 & $1.03(0.96-1.10)$ & 0.413 & 1.03(0.97-1.11) & 0.338 & 1.09(0.99-1.19) \\
\hline 7.0 & 1702(30.26) & reference & & reference & & reference & & reference \\
\hline 8.0 & 6147(29.88) & $1.01(0.96-1.06)$ & 0.730 & $1.05(0.99-1.11)$ & 0.094 & $1.04(0.98-1.10)$ & 0.174 & $1.09(1.02-1.18)$ \\
\hline$\geq 9$ & 188(31.60) & $1.06(0.91-1.23)$ & 0.445 & $1.07(0.92-1.24)$ & 0.397 & 1.07(0.92-1.24) & 0.406 & $1.08(0.89-1.31)$ \\
\hline \multicolumn{9}{|l|}{ LDL-C } \\
\hline$\leq 5$ & 405(19.46) & $1.03(0.92-1.15)$ & 0.643 & $1.02(0.91-1.14)$ & 0.768 & $1.04(0.93-1.17)$ & 0.515 & $1.07(0.93-1.23)$ \\
\hline 6.0 & 1031(19.14) & $1.03(0.94-1.12)$ & 0.540 & $1.02(0.94-1.12)$ & 0.584 & $1.03(0.94-1.12)$ & 0.532 & $1.08(0.98-1.21)$ \\
\hline 7.0 & 1035(18.40) & reference & & reference & & reference & & reference \\
\hline 8.0 & $4269(20.75)$ & $1.18(1.10-1.26)$ & $<0.001$ & $1.20(1.12-30)$ & $<0.001$ & $1.21(1.12-1.30)$ & $<0.001$ & $1.25(1.14-1.36)$ \\
\hline$\geq 9$ & $100(16.81)$ & $1.00(0.81-1.22)$ & 0.969 & $0.99(0.81-1.22)$ & 0.952 & $1.00(0.81-1.23)$ & 0.985 & $0.88(0.67-1.14)$ \\
\hline \multicolumn{9}{|l|}{ HDL-C } \\
\hline$\leq 5$ & 1482(71.22) & $1.01(0.95-1.07)$ & 0.779 & $1.01(0.95-1.08)$ & 0.655 & $1.02(0.96-1.08)$ & 0.605 & $1.02(0.95-1.09)$ \\
\hline 6.0 & $3658(67.90)$ & $1.01(0.97-1.06)$ & 0.570 & $1.02(0.97-1.06)$ & 0.505 & $1.01(0.97-1.06)$ & 0.579 & $1.02(0.96-1.07)$ \\
\hline 7.0 & $3638(64.69)$ & reference & & reference & & reference & & reference \\
\hline 8.0 & $13,610(66.15)$ & $1.11(1.07-1.16)$ & $<0.001$ & $1.11(1.07-1.15)$ & $<0.001$ & $1.10(1.06-1.15)$ & $<0.001$ & $1.11(1.06-1.16)$ \\
\hline$\geq 9$ & $412(69.24)$ & $1.27(1.15-1.41)$ & $<0.001$ & $1.27(1.14-1.40)$ & $<0.001$ & 1.24(1.12-1.38) & $<0.001$ & $1.21(1.07-1.35)$ \\
\hline \multicolumn{9}{|c|}{ Dyslipidemia } \\
\hline$\leq 5$ & $1298(62.37)$ & $0.96(0.90-1.02)$ & 0.166 & $0.96(0.90-1.02)$ & 0.955 & $0.98(0.92-1.04)$ & 0.471 & $0.99(0.92-1.08)$ \\
\hline 6.0 & $3558(66.05)$ & $1.04(0.99-1.10)$ & 0.061 & $1.04(0.99-1.10)$ & 0.061 & $1.05(1.00-1.10)$ & 0.036 & $1.07(1.01-1.14)$ \\
\hline 7.0 & $3695(65.70)$ & reference & & reference & & reference & & reference \\
\hline 8.0 & $13,081(63.58)$ & $0.95(0.92-0.99)$ & 0.012 & $1.01(0.97-1.05)$ & 0.564 & $1.01(0.97-1.05)$ & 0.509 & $1.08(1.02-1.14)$ \\
\hline$\geq 9$ & $379(63.70)$ & 0.98(0.88-1.09) & 0.681 & 0.98(0.88-1.09) & 0.706 & 0.98(0.88-1.09) & 0.685 & $1.08(0.95-1.24)$ \\
\hline
\end{tabular}

Model 1 adjusted for age and sex

Model 2 was further adjusted for level of education, smoking, alcohol, physical activity, and snoring. Model 3 was further adjusted for hypertension, diabetes mellitus, body mass index, and high-sensitivity C-reactive protein

${ }^{a}$ Adjusted for model 3 and further excluded individuals with transient changes in lipid profile (e.g., reversion from dyslipidemia to normal blood lipid)

level of h-CRP was higher in participants with dyslipidemia than in those without dyslipidemia (Table 2).

\section{Study strengths and limitations}

The major strengths of the current study include its large sample size and the availability of information on potential confounders and mediators. However, this study has some limitations. First, sleep duration data were collected through self-reported questionnaires. The use of more precise measures of sleep-related variables, such as polysomnographies, may reduce variability; however, these techniques are logistically unfeasible to perform in large epidemiological studies such as ours. Second, data on sleep quality, such as difficulty falling asleep, excessive daytime sleepiness, and obstructive sleep apnea (OSA), were not collected in the current study. A very recent study has shown that the association between OSA and impaired lipid profiles is partly determined genetically [37]. However, snoring status was adjusted as an alternative confounder since patients with OSA often have symptoms of snoring status. Third, some time-varying variables, such as lipid-lowering 


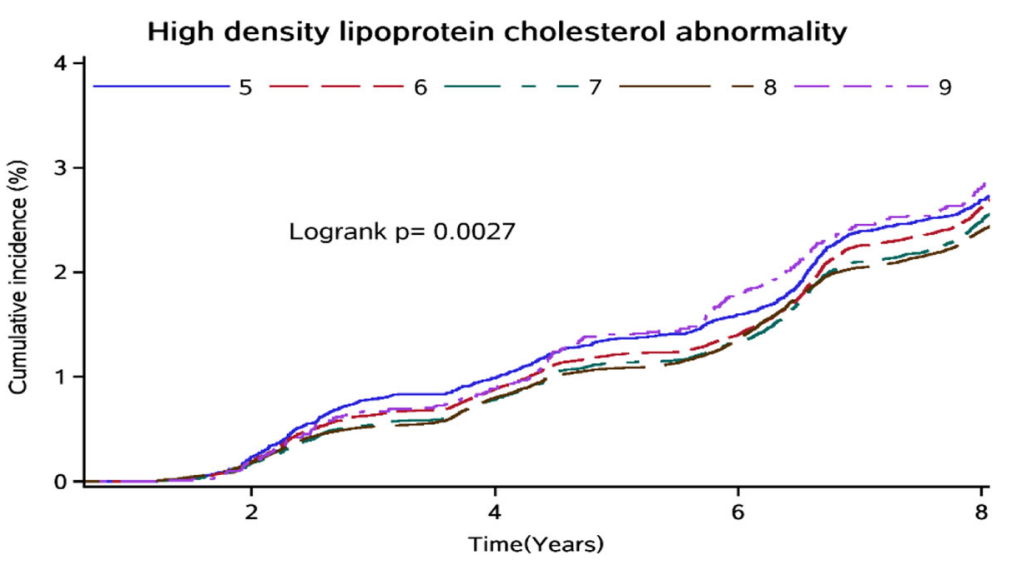

Fig. 2 Cumulative incidence of high-density lipoprotein cholesterol abnormality among Kailuan Study participants in different sleep duration groups

Table 4 Model 3 results on the associations between sleep duration and abnormal serum lipid levels stratified by sex and age

\begin{tabular}{|c|c|c|c|c|c|c|c|c|}
\hline \multirow{2}{*}{$\begin{array}{l}\text { Sleep } \\
\text { duration } \\
\text { (h) }\end{array}$} & \multicolumn{2}{|l|}{ Women } & \multicolumn{2}{|l|}{ Men } & \multicolumn{2}{|l|}{$\underline{\text { Age }<60}$} & \multicolumn{2}{|l|}{ Age $\geq 60$} \\
\hline & HR (95\% CI) & $\boldsymbol{P}$ value & HR $(95 \%$ Cl) & $\boldsymbol{P}$ value & HR $(95 \%$ Cl) & $\boldsymbol{P}$ value & HR (95\% Cl) & $\boldsymbol{P}$ value \\
\hline \multicolumn{9}{|l|}{ TC } \\
\hline$\leq 5$ & $1.04(0.89-1.23)$ & 0.596 & $0.95(0.85-1.06)$ & 0.135 & $1.01(0.92-1.10)$ & 0.868 & $0.88(0.75-1.05)$ & 0.156 \\
\hline 6.0 & $1.07(0.95-1.21)$ & 0.269 & $1.01(0.99-1.12)$ & 0.690 & $1.02(0.96-1.08)$ & 0.530 & $0.95(0.83-1.09)$ & 0.478 \\
\hline 7.0 & reference & & reference & & reference & & reference & \\
\hline 8.0 & $0.98(0.89-1.07)$ & 0.600 & $1.05(0.99-1.12)$ & 0.737 & $0.99(0.94-1.04)$ & 0.670 & $1.07(0.94-1.20)$ & 0.307 \\
\hline$\geq 9$ & $1.04(0.82-1.33)$ & 0.724 & $1.08(0.91-1.29)$ & 0.270 & $0.97(0.84-1.12)$ & 0.694 & $0.88(0.61-1.25)$ & 0.464 \\
\hline \multicolumn{9}{|l|}{ TG } \\
\hline$\leq 5$ & $1.11(0.89-1.40)$ & 0.349 & $0.95(0.85-1.06)$ & 0.338 & $1.04(0.94-1.16)$ & 0.424 & $0.73(0.56-0.96)$ & 0.022 \\
\hline 6.0 & $1.14(0.96-1.34)$ & 0.134 & $1.01(0.94-1.09)$ & 0.735 & $1.04(0.97-1.12)$ & 0.297 & $0.99(0.81-1.21)$ & 0.941 \\
\hline 7.0 & reference & & reference & & reference & & reference & \\
\hline 8.0 & $1.08(0.95-1.23)$ & 0.224 & $1.05(0.99-1.12)$ & 0.105 & $1.05(0.99-1.12)$ & 0.090 & $1.00(0.84-1.21)$ & 0.920 \\
\hline$\geq 9$ & $1.21(0.88-1.67)$ & 0.238 & $1.08(0.91-1.29)$ & 0.388 & $1.08(0.92-1.27)$ & 0.339 & $1.11(0.70-1.76)$ & 0.664 \\
\hline \multicolumn{9}{|l|}{ LDL-C } \\
\hline$\leq 5$ & $1.30(1.01-1.67)$ & 0.039 & $0.98(0.86-1.11)$ & 0.710 & $1.17(1.03-1.33)$ & 0.019 & $0.77(0.60-1.00)$ & 0.047 \\
\hline 6.0 & $1.09(0.90-1.34)$ & 0.380 & $1.01(0.92-1.11)$ & 0.856 & $1.06(0.96-1.16)$ & 0.257 & $0.93(0.76-1.14)$ & 0.492 \\
\hline 7.0 & reference & & reference & & reference & & reference & \\
\hline 8.0 & $1.33(1.15-1.55)$ & $<0.001$ & $1.17(1.08-1.27)$ & $<0.001$ & $1.21(1.12-1.31)$ & $<0.001$ & $1.25(1.05-1.48)$ & 0.013 \\
\hline$\geq 9$ & $1.26(0.86-1.84)$ & 0.232 & $0.94(0.73-1.20)$ & 0.600 & $1.05(0.84-1.31)$ & 0.668 & $0.81(0.48-1.37)$ & 0.427 \\
\hline \multicolumn{9}{|l|}{ HDL-C } \\
\hline$\leq 5$ & $0.99(0.85-1.16)$ & 0.921 & $1.02(0.96-1.09)$ & 0.511 & $1.01(0.94-1.09)$ & 0.782 & $1.03(0.92-1.15)$ & 0.624 \\
\hline 6.0 & $1.03(0.92-1.16)$ & 0.633 & $1.01(0.96-1.06)$ & 0.630 & $1.02(0.97-1.08)$ & 0.396 & $0.97(0.88-1.07)$ & 0.588 \\
\hline 7.0 & reference & & reference & & reference & & reference & \\
\hline 8.0 & $1.06(0.97-1.16)$ & 0.178 & $1.10(1.06-1.15)$ & $<0.001$ & $1.10(1.05-1.15)$ & $<0.001$ & $1.11(1.02-1.21)$ & $<0.05$ \\
\hline$\geq 9$ & $1.14(0.91-1.44)$ & 0.249 & $1.25(1.12-1.41)$ & $<0.001$ & $1.24(1.10-1.39)$ & $<0.001$ & $1.12(0.90-1.40)$ & 0.317 \\
\hline \multicolumn{9}{|c|}{ Dyslipidemia } \\
\hline$\leq 5$ & $1.14(0.98-1.31)$ & 0.086 & $0.94(0.88-1.01)$ & 0.098 & $1.03(0.96-1.11)$ & 0.418 & $0.85(0.74-0.98)$ & 0.027 \\
\hline 6.0 & $1.15(1.01-1.28)$ & 0.010 & $1.03(0.98-1.09)$ & 0.243 & $1.07(1.02-1.12)$ & 0.011 & $0.99(0.89-1.11)$ & 0.902 \\
\hline 7.0 & reference & & reference & & reference & & reference & \\
\hline 8.0 & $1.03(0.95-1.12)$ & 0.452 & $1.03(0.98-1.07)$ & 0.245 & $1.01(0.97-1.05)$ & 0.700 & $1.10(0.99-1.22)$ & 0.062 \\
\hline$\geq 9$ & $1.13(0.92-1.39)$ & 0.253 & $0.97(0.85-1.10)$ & 0.587 & $1.02(0.91-1.14)$ & 0.763 & $0.89(0.67-1.18)$ & 0.427 \\
\hline
\end{tabular}

Model 3 was adjusted for age (stratified by sex), sex (stratified by age), education, smoking, alcohol, physical activity, snoring, hypertension, diabetes mellitus, body mass index, and C-reactive protein 
therapy, physical activity, smoking habits, daily alcohol consumption, onset of systemic diseases during the period, and statins medication use at each follow-up, may modify serum lipids levels or sleep duration and have a favorable effect on the current results. For example, statins have a well-known side effect of tiredness and fatigue, which may result in long sleep times $[38,39]$. Paradoxically, patients who take statins may have better lipid profiles but long sleep times. Fourth, the proportion of individuals in the "9 or more hours of sleep" category is very small compared with the proportions in other categories. Thus, the estimates in this group might be unstable and influenced by employment status. Fifth, as participants were recruited from Tangshan (an industrial city located in northern China), the cohort is not nationally representative, and the generalizability of the current results to other geographic regions and to people of other racial/ethnic groups is not known. Finally, the current study was an observational study and we investigated only the association between baseline sleep duration and risk of dyslipidemia; thus, we did not consider changes at each time point in sleep duration. Indeed, any subsequent alterations in sleep duration may lead to a nondifferential misclassification and potential underestimation of the sleep-dyslipidemia association.

\section{Conclusions}

In conclusion, the results of this study indicate that long sleep duration is associated with a low HDL-C level among the Kailuan community population. The amount of sleep might play a key role in the risk of future lipid profile abnormalities. Encouraging and supporting individuals to pursue $7 \mathrm{~h}$ of sleep per night may have significant beneficial effects towards stemming the growing incidence of dyslipidemia in China.

\section{Abbreviations}

LDL-C: Low-density lipoprotein cholesterol; HDL-C: High-density lipoprotein cholesterol; CVD: Cardiovascular disease; TC: Total cholesterol;

TG: Triglyceride; BMI: Body mass index; FBG: Fasting blood glucose; hCRP: High-sensitivity C-reactive protein; HR: Hazard ratio

\section{Acknowledgements}

We appreciate the help and support from all participants and their relatives who took part in the study and the members of the survey teams from the Kailuan community.

\section{Authors' contributions}

S.W. and X.W. conceived and designed this study; X.L. directed the data analysis, X.L. and Q.S. wrote the paper; W.Z. prepared the database and reviewed the paper; X.W. and S.W. conducted the quality assurance and reviewed and edited the paper. All authors reviewed the manuscript. The author(s) read and approved the final manuscript.

\section{Funding}

No funding was received.

\section{Availability of data and materials}

The datasets used and/or analyzed during the current study are not available.

\section{Ethics approval and consent to participate}

The study was approved by the Ethics Committee of Kailuan General Hospital in compliance with the Declaration of Helsinki, and all participants provided written informed consent.

\section{Consent for publication}

Not applicable.

\section{Competing interests}

The authors declare that they have no competing interests.

Received: 20 February 2020 Accepted: 10 August 2020

Published online: 15 August 2020

\section{References}

1. Makino S, Hirose S, Kakutani M, Fujiwara M, Nishiyama M, Terada Y, Ninomiya H. Association between nighttime sleep duration, midday naps, and glycemic levels in Japanese patients with type 2 diabetes. Sleep Med. 2018:44:4-11.

2. Byrne EM, Gehrman PR, Trzaskowski M, Tiemeier H, Pack Al. Genetic correlation analysis suggests association between increased self-reported sleep duration in adults and schizophrenia and type 2 diabetes. Sleep. 2016; 39:1853-7.

3. Gangwisch JE, Heymsfield SB, Boden-Albala B, Buijs RM, Kreier F, Pickering TG, Rundle AG, Zammit GK, Malaspina D. Sleep duration as a risk factor for diabetes incidence in a large U.S. sample. Sleep. 2007;30:1667-73.

4. Miller MA, Kruisbrink M, Wallace J, Ji C, Cappuccio FP. Sleep duration and incidence of obesity in infants, children, and adolescents: a systematic review and meta-analysis of prospective studies. Sleep. 2018;41(4). https:// doi.org/10.1093/sleep/zsy018.

5. Wang F, Liu H, Wan Y, Li J, Chen Y, Zheng J, Huang T, Li D. Sleep duration and overweight/obesity in preschool-aged children: a prospective study of up to 48,922 children of the Jiaxing birth cohort. Sleep. 2016;39:2013-9.

6. Kim CW, Chang Y, Kang JG, Ryu S. Changes in sleep duration and subsequent risk of hypertension in healthy adults. Sleep. 2018;41. https:// doi.org/10.1093/sleep/zsy159.

7. Shivashankar R, Kondal D, Ali MK, Gupta R, Pradeepa R, Mohan V, Kadir MM, Narayan KMV, Tandon N, Prabhakaran D, Peasey A. Associations of sleep duration and disturbances with hypertension in metropolitan cities of Delhi, Chennai, and Karachi in South Asia: cross-sectional analysis of the CARRS study. Sleep. 2017;40(9):Zsx119.

8. Bertisch SM, Pollock BD, Mittleman MA, Buysse DJ, Bazzano LA, Gottlieb DJ, Redline S. Insomnia with objective short sleep duration and risk of incident cardiovascular disease and all-cause mortality: sleep heart health study. Sleep. 2018;41(6):zsy047.

9. Hoevenaar-Blom MP, Spijkerman AM, Kromhout D, van den Berg JF, Verschuren WM. Sleep duration and sleep quality in relation to 12-year cardiovascular disease incidence: the MORGEN study. Sleep. 2011;34:148792.

10. Tsai TC, Wu JS, Yang YC, Huang YH, Lu FH, Chang CJ. Long sleep duration associated with a higher risk of increased arterial stiffness in males. Sleep. 2014;37:1315-20.

11. Kaneita Y, Uchiyama M, Yoshiike N, Ohida T. Associations of usual sleep duration with serum lipid and lipoprotein levels. Sleep. 2008;31: $645-52$.

12. Kinuhata S, Hayashi T, Sato KK, Uehara S, Oue K, Endo G, Kambe H, Fukuda K. Sleep duration and the risk of future lipid profile abnormalities in middle-aged men: the Kansai healthcare study. Sleep Med. 2014:15:1379-85.

13. Kong AP, Wing YK, Choi KC, Li AM, Ko GT, Ma RC, Tong PC, Ho CS, Chan $\mathrm{MH}, \mathrm{Ng} \mathrm{MH}$, et al. Associations of sleep duration with obesity and serum lipid profile in children and adolescents. Sleep Med. 2011; 12:659-65.

14. Lin PMD, Chang KT, Lin YA, Tzeng IS, Chuang HH, Chen JY. Association between self-reported sleep duration and serum lipid profile in a middleaged and elderly population in Taiwan: a community-based, cross-sectional study. BMJ Open. 2017;7:e015964. 
15. Petrov ME, Kim Y, Lauderdale D, Lewis CE, Reis JP, Carnethon MR, Knutson K, Glasser SJ. Longitudinal associations between objective sleep and lipids: the CARDIA study. Sleep. 2013;36:1587-95.

16. Shin HY, Kang G, Kim SW, Kim JM, Yoon JS, Shin IS. Associations between sleep duration and abnormal serum lipid levels: data from the Korean National Health and Nutrition Examination Survey (KNHANES). Sleep Med. 2016;24:119-23.

17. Sabanayagam C, Shankar A. Sleep duration and hypercholesterolaemia: results from the National Health Interview Survey 2008. Sleep Med. 2012;13: $145-50$.

18. Zhan Y, Chen R, Yu J. Sleep duration and abnormal serum lipids: the China health and nutrition survey. Sleep Med. 2014;15:833-9.

19. Williams CJ, Hu FB, Patel SR, Mantzoros CS. Sleep duration and snoring in relation to biomarkers of cardiovascular disease risk among women with type 2 diabetes. Diabetes Care. 2007;30:1233-40.

20. Bjorvatn B, Sagen IM, Oyane N, Waage S, Fetveit A, Pallesen S, Ursin $R$. The association between sleep duration, body mass index and metabolic measures in the Hordaland health study. J Sleep Res. 2007; 16:66-76.

21. Gangwisch JE, Malaspina D, Babiss LA, Opler MG, Posner K, Shen S, Turner JB, Zammit GK, Ginsberg HN. Short sleep duration as a risk factor for hypercholesterolemia: analyses of the national longitudinal study of adolescent health. Sleep. 2010;33:956-61.

22. Turer CB, Brady TM, de Ferranti SD. Obesity, hypertension, and dyslipidemia in childhood are key modifiable antecedents of adult cardiovascular disease: a call to action. Circulation. 2018:137:1256-9.

23. Tobert JA, Newman CB. Management of dyslipidemia for cardiovascular disease risk reduction. Ann Intern Med. 2016;164:509.

24. Smiley A, King D, Harezlak J, Dinh P, Bidulescu A. The association between sleep duration and lipid profiles: the NHANES 2013-2014. J Diabetes Metab Disord. 2019;18:315-22

25. Song Q, Liu X, Hu W, Zhou W, Liu A, Wang X, Wu S. Long sleep duration is an independent risk factor for incident atrial fibrillation in a Chinese population: a prospective cohort study. Sci Rep. 2017;7:3679.

26. Liu X, Song Q, Hu W, Han X, Gan J, Zheng X, Wang X, Wu S. Night sleep duration and risk of incident Anemia in a Chinese population: a prospective cohort study. Sci Rep. 2018;8:3975.

27. Zhang Q, Zhou Y, Gao X, Wang C, Zhang S, Wang A, Li N, Bian L, Wu J, Jia $Q$, et al. Ideal cardiovascular health metrics and the risks of ischemic and intracerebral hemorrhagic stroke. Stroke. 2013;44:2451-6.

28. Bachorik PS, Ross JW. National Cholesterol Education Program recommendations for measurement of low-density lipoprotein cholesterol: executive summary. The National Cholesterol Education Program Working Group on Lipoprotein Measurement. Clin Chem. 1995;41:1414-20.

29. Joint Committee for Developing Chinese guidelines on P, Treatment of Dyslipidemia in A. Chinese guidelines on prevention and treatment of dyslipidemia in adults. Zhonghua Xin Xue Guan Bing Za Zhi. 2007; 35:390-419.

30. Nakanishi N, Nakamura K, Ichikawa S, Suzuki K, Tatara K. Relationship between lifestyle and serum lipid and lipoprotein levels in middle-aged Japanese men. Eur J Epidemiol. 1999:15:341-8.

31. Tan X, Chapman CD, Cedernaes J, Benedict C. Association between long sleep duration and increased risk of obesity and type 2 diabetes: a review of possible mechanisms. Sleep Med Rev. 2018;40:127-34.

32. Gottlieb DJ, Punjabi NM, Newman AB, Resnick HE, Redline S, Baldwin CM, Nieto FJ. Association of sleep time with diabetes mellitus and impaired glucose tolerance. Arch Intern Med. 2005;165:863-7.

33. Ayas NT, White DP, Al-Delaimy WK, Manson JE, Stampfer MJ, Speizer FE, Patel S, Hu FB. A prospective study of self-reported sleep duration and incident diabetes in women. Diabetes Care. 2003;26:380-4.

34. Gepner AD, Piper ME, Johnson HM, Fiore MC, Baker TB, Stein JH. Effects of smoking and smoking cessation on lipids and lipoproteins: outcomes from a randomized clinical trial. Am Heart J. 2011;161:145-51.

35. Palmer CD, Harrison GA, Hiorns RW. Association between smoking and drinking and sleep duration. Ann Hum Biol. 1980;7:103-7.

36. Nam GE, Cho KH, Park YG, Han KD, Choi YS, Kim SM, Lee KS, Ko BJ, Kim YH, Han BD, Kim DH. Socioeconomic status and dyslipidemia in Korean adults: the 2008-2010 Korea National Health and nutrition examination survey. Prev Med. 2013;57:304-9.

37. Meszaros M, Tarnoki AD, Tarnoki DL, Kovacs DT, Forgo B, Lee J, Sung J, Vestbo J, Muller V, Kunos L, Bikov A. Obstructive sleep apnea and hypertriglyceridaemia share common genetic background: Results of a twin study. J Sleep Res. 2020:e12979. https://doi.org/10.1111/jsr.12979.

38. Gonzalez M, Macias-Escalada E, Cobo J, Fernandez Mondragon MP, GomezMoreno G, Martinez-Martinez M, de Carlos F. Can treatment with statins have a negative influence on the tolerance of mandibular advancement devices? Sleep Breath. 2016;20:1363-6.

39. Vrablik M, Catapano AL, Wiklund O, Qian Y, Rane P, Grove A, Martin ML. Understanding the patient perception of statin experience: a qualitative study. Adv Ther. 2019;36:2723-43.

\section{Publisher's Note}

Springer Nature remains neutral with regard to jurisdictional claims in published maps and institutional affiliations.
Ready to submit your research? Choose BMC and benefit from:

- fast, convenient online submission

- thorough peer review by experienced researchers in your field

- rapid publication on acceptance

- support for research data, including large and complex data types

- gold Open Access which fosters wider collaboration and increased citations

- maximum visibility for your research: over $100 \mathrm{M}$ website views per year

At BMC, research is always in progress.

Learn more biomedcentral.com/submissions 